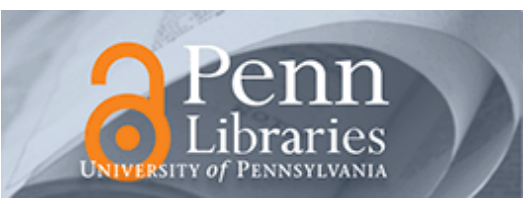

University of Pennsylvania

ScholarlyCommons

8-2015

\title{
Robust Monitoring of Hypovolemia in Intensive Care Patients Using Photoplethysmogram Signals
}

\author{
Alexander Roederer \\ University of Pennsylvania, roederer@seas.upenn.edu \\ James Weimer \\ University of Pennsylvania, weimerj@seas.upenn.edu \\ Joseph Dimartino \\ University of Pennsylvania, joseph.dimartino@uphs.upenn.edu \\ Jacob Gutsche \\ University of Pennsylvania, jacob.gutsche@uphs.upenn.edu \\ Insup Lee

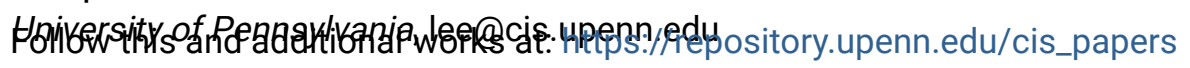 \\ Part of the Analytical, Diagnostic and Therapeutic Techniques and Equipment Commons, Computer \\ Engineering Commons, Computer Sciences Commons, and the Other Medicine and Health Sciences \\ Commons
}

\section{Recommended Citation}

Alexander Roederer, James Weimer, Joseph Dimartino, Jacob Gutsche, and Insup Lee, "Robust Monitoring of Hypovolemia in Intensive Care Patients Using Photoplethysmogram Signals", 37th Annual International Conference of the IEEE Engineering in Medicine and Biology Society (EMBC 2015). August 2015.

http://dx.doi.org/10.1109/EMBC.2015.7318656

37th Annual International Conference of the IEEE Engineering in Medicine and Biology Society (EMBC 2015). Milan, Italy, August 25-29, 2015.

http://emb.citengine.com/event/embc-2015/paper-details?pdID=4497

Preliminary version of this paper is available at http://repository.upenn.edu/cis_papers/781/

This paper is posted at ScholarlyCommons. https://repository.upenn.edu/cis_papers/787

For more information, please contact repository@pobox.upenn.edu. 


\title{
Robust Monitoring of Hypovolemia in Intensive Care Patients Using Photoplethysmogram Signals
}

\author{
Abstract \\ The paper presents a fingertip photoplethysmography based technique to assess patient fluid status that \\ is robust to waveform artifacts and health variability in the underlying patient population. The technique is \\ intended for use in intensive care units, where patients are at risk for hypovolemia, and signal artifacts \\ and inter-patient variations in health are common. Input signals are preprocessed to remove artifact, then \\ a parameter-invariant statistic is calculated to remove effects of patient-specific physiology. Patient data \\ from the Physionet MIMICII database was used to evaluate the performance of this technique. The \\ proposed method was able to detect hypovolemia within 24 hours of onset in all hypovolemic patients \\ tested, while producing minimal false alarms over non-hypovolemic patients.

\section{Disciplines} \\ Analytical, Diagnostic and Therapeutic Techniques and Equipment | Computer Engineering | Computer \\ Sciences | Other Medicine and Health Sciences

\section{Comments} \\ 37th Annual International Conference of the IEEE Engineering in Medicine and Biology Society (EMBC \\ 2015). Milan, Italy, August 25-29, 2015. \\ http://emb.citengine.com/event/embc-2015/paper-details?pdID=4497 \\ Preliminary version of this paper is available at http://repository.upenn.edu/cis_papers/781/
}




\title{
Robust Monitoring of Hypovolemia in Intensive Care Patients using Photoplethysmogram Signals
}

\author{
Alexander Roederer ${ }^{1}$, James Weimer ${ }^{1}$, Joseph DiMartino ${ }^{2}$, Jacob Gutsche ${ }^{2}$, and Insup Lee ${ }^{1}$
}

\begin{abstract}
The paper presents a fingertip photoplethysmography based technique to assess patient fluid status that is robust to waveform artifacts and health variability in the underlying patient population. The technique is intended for use in intensive care units, where patients are at risk for hypovolemia, and signal artifacts and inter-patient variations in health are common. Input signals are preprocessed to remove artifact, then a parameter-invariant statistic is calculated to remove effects of patient-specific physiology. Patient data from the Physionet MIMICII database was used to evaluate the performance of this technique. The proposed method was able to detect hypovolemia within 24 hours of onset in all hypovolemic patients tested, while producing minimal false alarms over non-hypovolemic patients.
\end{abstract}

\section{INTRODUCTION}

The photoplethysmograph (PPG) is an optical measurement used to changes in detect blood volume in the microvascular tissue bed. Devices (such as pulse oximeters) which measure PPG contain a light emitting diode and an optical sensor. Light is emitted into flesh and either reflected off bone and back to the sensor, or transmitted directly through the flesh and into the sensor. The amount of light reabsorbed by the sensor is impacted by the scattering, absorption, reflection, and fluorescence of the biological tissue [1].

PPG has seen widespread clinical application, as it is noninvasive and can be used to measure many different aspects of cardiovascular function, most commonly pulse rate and tissue oxygenation [1]. The signal also contains information about vascular distensibility, cardiac arrhythmia, systolic blood pressure, respiratory variability [2], and, notably, blood volume. Recorded pulses bear a direct relationship with perfusion, as larger blood volumes produce larger attenuation in the light source [1].

Patients who present to emergency rooms with trauma, patients undergoing surgery, and post-operative patients in intensive care units frequently suffer from hemorrhage. Persistent internal hemorrhage can, over time, cause a decrease in the volume of blood in the circulatory system, a condition known as hypovolemia [3]. Hypovolemia is common among post-operative patients. Bleeding-related complications (such as rapidly fatal hypovolemic shock) are a major cause of prolonged length of stay and death in hospitals [4], [5].

This research was supported in part by NSF CNS-1035715.

${ }^{1}$ Alexander Roederer, Dr. James Weimer, and Dr. Insup Lee are with the School of Engineering and Applied Science, University of Pennsylvania, 3330 Walnut Street, Philadelphia, Pennsylvania, USA. Email: [roederer|weimer $j \mid$ lee $]$ [ seas . upenn . edu

${ }^{2}$ Dr. Jacob Gutsche M.D. and Joseph DiMartino R.N., M.S.N. are with Penn Presbyterian Medical Center, University of Pennsylvania Health System, Philadelphia, Pennsylvania, USA. Dr. Jacob Gutsche is also with the Perelman School of Medicine, University of Pennsylvania.
Assessment of decreasing blood volume is one of the most difficult tasks in current clinical medicine [6], as the body's hemodynamic compensation mechanisms can mask changes in most of the vital signs which would traditionally be used to assess volume status. [3], [7], [8], [9], [10]. Patient fluid inputs and outputs are closely monitored for changes [11], as common medical practice holds that these changes may reflect changes in blood volume. These changes only occur, however, after significant blood is lost [12].

There have been numerous attempts to use the PPG waveform to noninvasively monitor fluid status and detect hypovolemia, with the goal of providing earlier, more accurate, less invasvie detection [12], [13], [14], [15], [16], [17], [18]. These studies have used compensatory reserve index (CRI), variations in pulse oximeter waveform amplitude $(\triangle \mathrm{POP})$ and/or pleth variability index (PVI) (Masimo, Irvine, CA). Results show promise, but predictive values seem to vary substantially between studies [19], [20], and few studies gauge performance in critically ill patients.

While PPGs contain large amounts of information about a patient's cardiovascular function, they can be difficult to use because they often experience large amounts of artifact. The PPG sensor is sensitive to movement and orientation against the skin and small shifts can significantly impact the measured intensity of light, and subsequently the accuracy of the data. Detection is made still more challenging by interpatient variability. Patients' blood volumes and compensatory mechanisms vary, and the body's response to blood loss varies based on the severity and location of the bleed. Both of these problems make utilizing PPG for fluid management a non-trivial problem.

In this work, we present a technique for utilizing PPG waveforms to monitor patients' fluid status, in particular for detecting hypovolemia. We apply preprocessing to remove artifact, then apply novel parameter invariance techniques to create a statistic that is invariant to common forms of signal noise. We evaluate the detector over a set of both hypovolemic and non-hypovolemic ICU patients from the Physionet MIMIC II database [21], [22].

\section{Methodology}

To design a robust detector, this work employs parameter invariant statistics to build CFAR detectors as described in [23], [24]. We utilize a sampled average of the PPG waveform over time to test for fluid loss. This section first addresses modeling the PPG waveform, the describes design of the proposed robust test, and finally provides a description of the algorithm used for PPG waveform pre-processing. 


\section{A. PPG Trend Modeling}

Following the methodology in [24] for designing parameter-invariant monitors, in this subsection we develop models representing the PPG trends under normal (null) and hypovolemic (event) scenarios. The PPG waveform is composed of a static DC signal related to the absorption of light by the non-blood components of the body (i.e. bone, muscle, skin, etc.) and a dynamic AC signal corresponding to the blood-related absorption. In [25], it is shown that immobilized healthy patients experiencing central blood volume loss have AC PPG signals which tend to decrease in amplitude and pulse width such that the average value of the PPG waveform, over the respiratory cycle, decreases.

Let $P P G(t)$ be the value of the PPG waveform at time $t \geq 0$, and let $\overline{P P G}(n)$ with $n \in \mathbb{N}$ represent the $n$-th sampled average of the PPG waveform over a time window of $T$ seconds. (Note that $\overline{P P G}(n)$ is a function of $P P G(t)$ over the domain $(n-1) T \leq t<n T)$ Then we can model the trend of $\overline{P P G}$ in a hypovolemic scenario as

$$
H_{1}: \overline{P P G}(k+1)=\alpha_{1} \overline{P P G}(k)+\beta_{1}+\sigma_{1} n(k)
$$

with $\beta_{1}>0$ and $0<\alpha_{1}<1$ proportional to the fluid loss volume and fluid loss rate parameters, respectively. $\sigma_{1}$ represents the variance of the noise. Due to varying patient physiology and condition, the parameters $\alpha_{1}, \beta_{1}, \sigma_{1}$ are unknown.

Intensive care patients are rarely immobilized and healthy, thus the average of the PPG waveform of non-hypovolemic patients tends to drift over time. Rather than attempt to model all possible physiological scenarios that explain drifts in the PPG waveform, we model $\overline{P P G}$ under non-hypovolemic conditions (the null hypothesis) as a Brownian motion,

$$
H_{0} \quad: \quad \overline{P P G}(k+1)=\overline{P P G}(k)+\sigma_{0} n(k)
$$

where $\sigma_{0} n(k) \sim \mathrm{N}\left[0, \sigma_{0}^{2}\right]$ denotes the input noise. Consistent with the event model in (1), the noise parameter $\sigma_{0}$ is unknown.

The models developed in this subsection utilize medical trends to describe the dynamics of the PPG mean. As discussed in [24], the parameter-invariant design approach only requires models which capture the general trend of the PPG signal, and need not be an accurate first-principles representation of the hemodynamics.

\section{B. Parameter-Invariant Test Design}

As described in the previous section, the parameters of the models (2) and (1) are unknown, and vary over each patient. Attempting to estimate these parameters directly would require a prohibitive amount of data. Instead, this subsection introduces a test to identify signal patterns indicative of hypovolemia that is invariant to the model parameter values (a parameter-invariant test). A one-sided test statistic is used to produce a sufficient statistic threshold test with a constant false alarm rate (CFAR).

To develop the statistic, we assume a testing window of $K$ samples and write $y(k)=\overline{P P G}(k)-\overline{P P G}(k-1)$ and rearrange to obtain a time-concatenated model under each hypothesis that can be written as

$$
\begin{aligned}
& H_{0}: \boldsymbol{y}_{k}=\sigma_{0} \boldsymbol{n} \\
& H_{1}: \boldsymbol{y}_{k}=\boldsymbol{f}_{k}\left(\alpha_{1}-1\right)+1 \beta_{1}+\sigma_{1} \boldsymbol{n}
\end{aligned}
$$

where

$$
\boldsymbol{y}_{k}=\left[\begin{array}{c}
y(k-K) \\
\vdots \\
y(k)
\end{array}\right] \text { and } \boldsymbol{f}_{k}=\left[\begin{array}{c}
\overline{P P G}(k-K-1) \\
\vdots \\
\overline{P P G}(k-1)
\end{array}\right] \text {. }
$$

We then construct a sufficient statistic for the hypothesis testing problem in (3) which is invariant to the effect of the unknown parameters as

$$
t\left(\boldsymbol{y}_{k}\right)=\frac{\mathbf{1}^{\top} \boldsymbol{P}_{k} \boldsymbol{y}_{k}}{\sqrt{\mathbf{1}^{\top} \boldsymbol{P}_{k} \mathbf{1}} \sqrt{\boldsymbol{y}_{k}^{\top} \boldsymbol{P}_{k}\left(\boldsymbol{I}-\frac{1}{K} \mathbf{1} \mathbf{1}^{\top}\right) \boldsymbol{P}_{k} \boldsymbol{y}_{k}}}
$$

where

$$
\boldsymbol{P}_{k}=\boldsymbol{I}-\frac{\boldsymbol{f}_{k} \boldsymbol{f}_{k}^{\top}}{\boldsymbol{f}_{k}^{\top} \boldsymbol{f}_{k}}
$$

In words, we design invariant to the effect of $\alpha$ by projecting onto the null space of $\boldsymbol{f}_{k}$ (i.e. multiplying by $\boldsymbol{P}_{k}$ ).

The sufficient statistic $t$ represents the ratio of the signal affected by $\beta_{1}$ to the signal unaffected by $\beta_{1}$ such that the scaling imposed by $\sigma_{i}$ is canceled between the numerator and denominator. ${ }^{1}$ This eliminates the effect of the noise parameter $\sigma_{i}$ under each hypothesis.

A threshold test $\phi$ is then employed to decide between the hypotheses:

$$
\phi\left(\boldsymbol{y}_{k}\right)= \begin{cases}H_{0} & \text { if } t\left(\boldsymbol{y}_{k}\right) \geq \eta \\ H_{1} & \text { else }\end{cases}
$$

$\phi\left(\boldsymbol{y}_{k}\right)$ is CFAR since the distribution of the statistic $t$ is invariant to the unknown parameters under the null hypothesis. A CFAR detector is desireable as it has a constant probability of deciding $H_{1}$ when $H_{0}$ is actually true, regardless of the unknown parameters.

\section{PPG Waveform Preprocessing}

The PPG waveform is known to contain artifact associated with movement, spontaneous breathing, clipping, and missing data. The removal of PPG waveform artifact is an open area of research [14], [20]. Consistent with [8], [12], [25] we observe that without artifacts, the dominant nonDC frequencies of the PPG waveform correspond to the fundamental frequency of the heart rate and its harmonic frequencies. As test in (7) only requires the sampled average PPG waveform, $\overline{P P G}$, we can employ this observation to generate the sampled average PPG waveform at each time step $k, \overline{P P G}(k)$, corresponding to a $T$ second time window by dividing the $T$ second window into $J$ sub-windows of equal length. We then perform a spectral analysis via the fourier transform of each sub-window. The sub-window's data is

\footnotetext{
${ }^{1}$ Due to space constraints, a detailed derivation is omitted, but follows closely the formulation in [23].
} 
only included in the sampled average if the maximum nonDC frequency in that sub-window is likely to correspond to the heart rate. In the event that too many sub windows do not meet the criteria, we treat the sampled average at that time as a missing measurement. Formally, this process is described in the following algorithm (assuming $\omega=$ $\exp \{-i 2 \pi / N\}$ and $N_{0}$ and $J_{0}$ correspond to the minimum heart rate frequency and minimum number of sub-windows which must be included in the average, respectively):

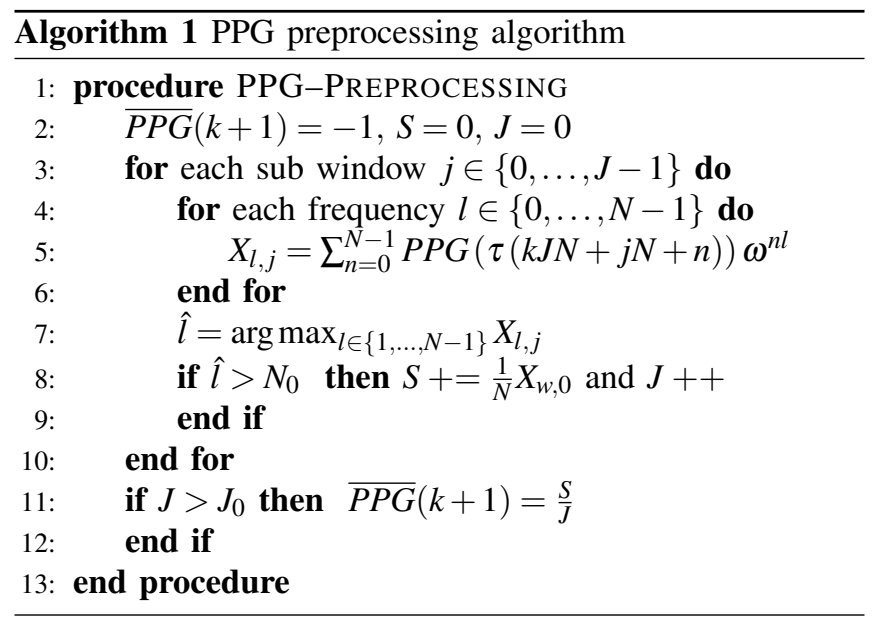

\section{RESUltS AND Discussion}

\section{A. Data used for Evaluation}

We evaluated the proposed technique over hypovolemic and non-hypovolemic patients drawn from the matched subset of the Physionet MIMIC II Waveform Database [21], [22]. The matched subset allowed us to use nursing notes to find annotated times of suspected hypovolemia and subsequent fluid administration. To select non-hypovolemic patients, we chose patients from the database who had PPG waveforms, did not die, and had four or fewer ICD9 codes.

To select patients with a high likelihood of hypovolemia, we searched for patients with documented hypovolemia ICD9 codes on discharge with accompanying notes documenting approximate time of suspected hypovolemia. From these patients, we selected those who did not die and who had available PPG waveforms. Time of hypovolemia was annotated as the timestamp of the note describing suspected hypovolemia in the patient's file. We ran the algorithm only on data from the ICU stay with documented hypovolemia. Probability of false alarm was set to 4 false alarms per day.

\section{B. Parameter Invariant Results}

The results of running the proposed technique on each patient's PPG data can be found in Table I. For all seven hypovolemic patients, our detector presented a higher-thanaverage number of alarms within a 24 hour envelope of first documented hypovolemia. ${ }^{2}$ A number of the hypovolemic patients had alarms prior to documented suspicion of hypovolemia. Several also had alarms long after. We consider these other alarms inconclusive, as no clear indication of when hypovolemia ended for these patients. Total alarm duration for these seven patients was 189 minutes (3.15 hours) out of a total time in the ICU of 474.81 hours (19.8 days).

The detector generated alarms for only three of the 18 non-hypovolemic patients. In total, these patients had a total 388.18 hours of ICU time, and experienced 18 alarms. These alarms had a duration of 196.8 minutes (3.28 hours). One of these patients (patient 19608) suffered respiratory issues which we believe may have triggered the false alarms.

\section{CONCLUSION}

We have described a method for creating a hypovolemia detector robust to artifact and noise by employing parameter invariant statistical techniques to PPG waveforms. Preliminary tests on retrospective patient data suggest the proposed detector produces alarms near and before time of diagnosed hypovolemia while producing few false alarms in healthy patients, and seems to perform better than PVI, a state of the art method, over these patients. The proposed approach seems to perform well over noisy data with artifact, making it particularly applicable to clinical intensive care settings.

Though the size of the patient data set was too small to assess definitive sensitivity or specificity, the results are promising. We hope to further expand the data set to provide a more robust assessment of the technique. To improve the technique's predictive power, future work will investigate extracting more features from the PPG waveform and applying parameter invariance, as well as the possibility of using other waveforms to improve preprocessing artifact detection.

\section{REFERENCES}

[1] J. Allen, "Photoplethysmography and its application in clinical physiological measurement." Physiological measurement, vol. 28, no. 3, pp. R1-39, Mar. 2007.

[2] K. H. Shelley, "Photoplethysmography: beyond the calculation of arterial oxygen saturation and heart rate." Anesthesia and analgesia, vol. 105, no. 6 Suppl, pp. S31-6, tables of contents, Dec. 2007.

[3] G. Gutierrez, H. D. Reines, and M. E. Wulf-Gutierrez, "Clinical review: Hemorrhagic Shock," Critical care (London, England), vol. 8, no. 5, pp. 373-81, Oct. 2004.

[4] M. E. Stokes, X. Ye, M. Shah, K. Mercaldi, M. W. Reynolds, M. F. T. Rupnow, and J. Hammond, "Impact of bleeding-related complications and/or blood product transfusions on hospital costs in inpatient surgical patients." BMC health services research, vol. 11, no. 1, p. 135, Jan. 2011.

[5] M. Moscucci, K. Fox, C. P. Cannon, W. Klein, J. Lopez-Sendon, G. Montalescot, K. White, and R. Goldberg, "Predictors of major bleeding in acute coronary syndromes: the Global Registry of Acute Coronary Events (GRACE)," European Heart Journal, vol. 24, no. 20, pp. 1815-1823, Oct. 2003.

[6] P. E. Marik, R. Cavallazzi, T. Vasu, and A. Hirani, "Dynamic changes in arterial waveform derived variables and fluid responsiveness in mechanically ventilated patients: a systematic review of the literature." Critical care medicine, vol. 37, no. 9, pp. 2642-7, Sep. 2009.

${ }^{2}$ We felt a 24 hour envelope was reasonable, as in many patients gradual volume loss is not traditionally apparent for several hours or days [26]. No standard currently exists for the acceptable maximum amount of time to alert care teams to hypovolemia. For gradual volume loss, clinicians currently expect longer time to detection, as they frequently depend on non-urgent detection methods such as postural hypotension or low urine output [27]. 


\begin{tabular}{|c|c|c|c|c|c|c|}
\hline Patient & $\begin{array}{l}\text { Length of } \\
\text { ICU Stay } \\
\text { (Hours) }\end{array}$ & $\begin{array}{l}\text { Percentage } \\
\text { of "good" } \\
\text { PPG data }\end{array}$ & Hypovolemic? & $\begin{array}{l}\text { Number of } \\
\text { Alarms }\end{array}$ & $\begin{array}{l}\text { Mean Alarm } \\
\text { Length } \\
\text { (Minutes) }\end{array}$ & $\begin{array}{c}\text { Alarms within } 24 \text { hours } \\
\text { of onset }\end{array}$ \\
\hline 03617 & 34.6 & 28.4 & No & 0 & $\mathrm{NaN}$ & N/A \\
\hline 03640 & 22.0 & 6.4 & No & 0 & $\mathrm{NaN}$ & N/A \\
\hline 05345 & 21.4 & 85.4 & No & 0 & $\mathrm{NaN}$ & N/A \\
\hline 08949 & 34.5 & 90.3 & No & 5 & 17.8 & N/A \\
\hline 11622 & 14.8 & 75.7 & No & 0 & $\mathrm{NaN}$ & N/A \\
\hline 11727 & 24.3 & 38.4 & No & 0 & $\mathrm{NaN}$ & N/A \\
\hline 14251 & 25.4 & 26.0 & No & 0 & $\mathrm{NaN}$ & N/A \\
\hline 19309 & 6.3 & 67.2 & No & 0 & $\mathrm{NaN}$ & N/A \\
\hline 19608 & 32.9 & 90.7 & No & 11 & 10.5 & N/A \\
\hline 21986 & 18.0 & 74.1 & No & 0 & $\mathrm{NaN}$ & N/A \\
\hline 27539 & 12.4 & 28.7 & No & 0 & $\mathrm{NaN}$ & N/A \\
\hline 28706 & 40.8 & 41.2 & No & 1 & 6.0 & N/A \\
\hline 29116 & 4.7 & 24.9 & No & 0 & $\mathrm{NaN}$ & N/A \\
\hline 29126 & 4.7 & 76.2 & No & 0 & $\mathrm{NaN}$ & N/A \\
\hline 30243 & 55.7 & 26.4 & No & 0 & $\mathrm{NaN}$ & N/A \\
\hline 31015 & 5.2 & 25.7 & No & 0 & $\mathrm{NaN}$ & N/A \\
\hline 31140 & 8.1 & 96.1 & No & 0 & $\mathrm{NaN}$ & N/A \\
\hline 32249 & 22.3 & 11.1 & No & 0 & $\mathrm{NaN}$ & N/A \\
\hline 00618 & 68.5 & 91.2 & Yes & 7 & 6.6 & 1 \\
\hline 06085 & 37.6 & 53.9 & Yes & 1 & 1.0 & 1 \\
\hline 07251 & 54.0 & 87.2 & Yes & 5 & 6.0 & 4 \\
\hline 12351 & 160.8 & 62.9 & Yes & 7 & 6.4 & 4 \\
\hline 16139 & 117.5 & 30.4 & Yes & 4 & 4.5 & 3 \\
\hline 17582 & 11.6 & 90.3 & Yes & 1 & 8.0 & 1 \\
\hline 22585 & 24.8 & 55.5 & Yes & 1 & 61.0 & 1 \\
\hline
\end{tabular}

TABLE I: Summary of patients selected from the Physionet MIMIC II database and results of the application of the proposed detector on these patients. Patient IDs are from the matched subset. Length of stay includes relevant single ICU stay.

[7] V. a. Convertino, C. a. Rickards, K. G. Lurie, and K. L. Ryan, "Hyperventilation in Response to Progressive Reduction in Central Blood Volume to Near Syncope," Aviation, Space, and Environmental Medicine, vol. 80, no. 12, pp. 1012-1017, Dec. 2009.

[8] V. a. Convertino, S. L. Moulton, G. Z. Grudic, C. a. Rickards, C. Hinojosa-Laborde, R. T. Gerhardt, L. H. Blackbourne, and K. L. Ryan, "Use of advanced machine-learning techniques for noninvasive monitoring of hemorrhage." The Journal of trauma, vol. 71, no. 1 Suppl, pp. S25-32, Jul. 2011.

[9] E. Zöllei, V. Bertalan, A. Németh, P. Csábi, I. László, J. Kaszaki, and L. Rudas, "Non-invasive detection of hypovolemia or fluid responsiveness in spontaneously breathing subjects." BMC anesthesiology, vol. 13, no. 1, p. 40, Jan. 2013

[10] S. McGee, W. B. I. Abernethy, and D. L. Simel, "Is This Patient Hypovolemic?" Journal of the American Medical Association, vol. 281, no. 11, p. 1022, Mar. 1999.

[11] K. Bartels, R. H. Thiele, and T. J. Gan, "Rational fluid management in today's ICU practice," Critical Care, vol. 17, no. Suppl 1, pp. 2-7, 2013.

[12] C. L. Stewart, J. Mulligan, G. Z. Grudic, V. a. Convertino, and S. L. Moulton, "Detection of low-volume blood loss: compensatory reserve versus traditional vital signs." The journal of trauma and acute care surgery, vol. 77, no. 6, pp. 892-7; discussion 897-8, Dec. 2014.

[13] A. A. Alian, N. J. Galante, N. S. Stachenfeld, D. G. Silverman, and K. H. Shelley, "Impact of central hypovolemia on photoplethysmographic waveform parameters in healthy volunteers part 2: frequency domain analysis." Journal of clinical monitoring and computing, vol. 25, no. 6, pp. 387-96, Dec. 2011.

[14] M. Cannesson, O. Desebbe, P. Rosamel, B. Delannoy, J. Robin, O. Bastien, and J.-J. Lehot, "Pleth variability index to monitor the respiratory variations in the pulse oximeter plethysmographic waveform amplitude and predict fluid responsiveness in the operating theatre." British journal of anaesthesia, vol. 101, no. 2, pp. 200-6, Aug. 2008.

[15] P. Forget, F. Lois, and M. de Kock, "Goal-directed fluid management based on the pulse oximeter-derived pleth variability index reduces lactate levels and improves fluid management." Anesthesia and analgesia, vol. 111, no. 4, pp. 910-4, Oct. 2010.

[16] R. Sahni, "Noninvasive monitoring by photoplethysmography." Clinics in perinatology, vol. 39, no. 3, pp. 573-83, 2012.
[17] T. Loupec, H. Nanadoumgar, D. Frasca, F. Petitpas, L. Laksiri, D. Baudouin, B. Debaene, C. Dahyot-Fizelier, and O. Mimoz, "Pleth variability index predicts fluid responsiveness in critically ill patients." Critical Care Medicine, vol. 39, no. 2, pp. 294-299, Feb. 2011.

[18] R. Pizov, A. Eden, D. Bystritski, E. Kalina, A. Tamir, and S. Gelman, "Arterial and Plethysmographic Waveform Analysis in Anesthetized Patients with Hypovolemia," Anesthesiology, vol. 113, no. 1, pp. 8391, 2010.

[19] L. P. Antonsen and K. A. Kirkeboen, "Evaluation of fluid responsiveness: is photoplethysmography a noninvasive alternative?" Anesthesiology research and practice, Jan. 2012.

[20] M. Cannesson, M. Aboy, C. K. Hofer, and M. Rehman, "Pulse pressure variation: where are we today?" Journal of Clinical Monitoring and Computing, vol. 25, no. 1, pp. 45-56, Apr. 2010

[21] M. Saeed, M. Villarroel, A. T. Reisner, G. Clifford, L.-W. Lehman, G. Moody, T. Heldt, T. H. Kyaw, B. Moody, and R. G. Mark, "Multiparameter intelligent monitoring in intensive care ii (mimic-ii): a public-access intensive care unit database," Critical care medicine, vol. 39, no. 5, p. 952, 2011.

[22] A. L. Goldberger, L. A. Amaral, L. Glass, J. M. Hausdorff, P. C. Ivanov, R. G. Mark, J. E. Mietus, G. B. Moody, C.-K. Peng, and H. E. Stanley, "Physiobank, physiotoolkit, and physionet components of a new research resource for complex physiologic signals," Circulation, vol. 101, no. 23, pp. e215-e220, 2000.

[23] L. L. Scharf and C. Demeure, Statistical Signal Processing. AddisonWesley Publishing Company, 1991.

[24] J. Weimer, R. Ivanov, A. Roederer, S. Chen, and I. Lee, "Parameter invariant design of medical alarms," IEEE Design \& Test, submitted.

[25] S. P. McGrath, K. L. Ryan, S. M. Wendelken, C. a. Rickards, and V. a. Convertino, "Pulse oximeter plethysmographic waveform changes in awake, spontaneously breathing, hypovolemic volunteers." Anesthesia and analgesia, vol. 112, no. 2, pp. 368-74, Feb. 2011.

[26] M. Y. Shamir, L. Kaplan, R. S. Marans, D. Willner, and Y. Klein, "Urine flow is a novel hemodynamic monitoring tool for the detection of hypovolemia," Anesthesia \& Analgesia, vol. 112, no. 3, pp. 593596, 2011.

[27] R. N. Sladen, "Oliguria in the icu: systematic approach to diagnosis and treatment," Anesthesiology Clinics of North America, vol. 18, no. 4, pp. 739-752, 2000. 